\title{
Dieta cetogênica: uma estratégia eficiente no controle de peso corporal?
}

\author{
Ketogenic diet: an efficient strategy in body weight control? \\ Dieta cetogénica: ¿una estrategia eficaz para controlar el peso corporal?
}

\section{Resumo}

A obesidade é um problema de saúde pública mundial que favorece doenças crônicas não transmissíveis afetando a qualidade de vida e o risco de morte. Portanto é necessário combater essa epidemia através da perda de peso corporal. Dietas restritas em carboidrato são alvos de questionamentos sobre eficácia, mecanismo de ação e efeitos adversos. Sob essa definição existe um protocolo dietético que apresenta uma restrição severa em carboidratos, chamada dieta cetogênica que originalmente foi indicada no tratamento não-farmacológico da epilepsia, atualmente tem sido indicada para perda de peso sem ainda haver um consenso na literatura. O objetivo deste trabalho foi realizar uma revisão narrativa da literatura sobre a dieta cetogênica quanto sua eficiência na perda de peso, repercussões metabólicas, performance esportiva e efeitos colaterais. Foram selecionados 27 artigos científicos publicados nos últimos quatorze anos, disponíveis nos portais PubMed, Scielo, Google Acadêmico e BioMed. Em 24 trabalhos houve grupo controle. Em 19 artigos os participantes foram submetidos a protocolos sem restrições calóricas. Em todos os estudos foi constatada a redução de peso ponderal dos participantes. Após a revisão, considera-se que a dieta cetogênica é eficaz na perda de peso, melhora certos parâmetros bioquímicos, porém quando as calorias são controladas os resultados na perda de peso tendem a ser similares a uma dieta controle, embora haja diferenças na bioquímica sérica.

Palavras-chave: Baixo carboidrato; Cetogênica; Perda de peso.

\begin{abstract}
Obesity is a worldwide public health problem that favors chronic noncommunicable diseases that affect the quality of life and the risk of death. Therefore, it's necessary to combat this epidemic through loss of body weight. Carbohydrate restricted diets are the subject of questions about efficacy, mechanism of action and adverse effects. Under this definition there is a dietary protocol that has a severe restriction on carbohydrates, the ketogenic diet, which was originally indicated for the non-pharmacological treatment in epilepsy, has currently been indicated for weight loss without a
\end{abstract}


consensus in the literature. The objective of this work was to carry out a narrative review of literature on the ketogenic diet as to its efficiency in weight loss, metabolic repercussions, sports performance and side effects. Twenty-six scientific articles published in the last fourteen years available in the portals PubMed, Scielo, Google Academic, BioMed was selected. In 24 studies there was participation of control group. In 18 articles the participants were submitted to protocols without caloric restrictions. In all the studies, weight reduction of the participants was verified. After the review, it's considered that the ketogenic diet is effective in weight loss, improves certain biochemical parameters, but when the calories are controlled the results in weight loss tend to be similar to a control diet, although there are differences in serum biochemistry.

Keywords: Low carbohydrate; Ketogenic; Weight loss.

\section{Resumen}

La obesidad es un problema de salud pública mundial, que favorece las enfermedades crónicas no transmisibles que afectan la calidad de vida y el riesgo de muerte. Por tanto, es necesario combatir esta epidemia perdiendo peso corporal. Las dietas restringidas en carbohidratos son objeto de preguntas sobre eficacia, mecanismo de acción y efectos adversos. Bajo esta definición, existe un protocolo dietético que está severamente restringido en carbohidratos, llamado dieta cetogénica, que originalmente estaba indicado para el tratamiento no farmacológico en la epilepsia, actualmente se ha indicado para la pérdida de peso sin un consenso en la literatura. El objetivo de este trabajo fue realizar una revisión narrativa de la literatura sobre la dieta cetogénica en cuanto a su eficacia en la pérdida de peso, repercusiones metabólicas, rendimiento deportivo y efectos secundarios. Se seleccionaron veintiséis artículos científicos publicados en los últimos catorce años, disponibles en los portales PubMed, Scielo, Google Scholar y BioMed. En 24 estudios, participó un grupo de control. En 18 artículos, los participantes fueron sometidos a protocolos sin restricciones calóricas. En todos los estudios, se observó reducción de peso de los participantes. Tras la revisión, la dieta cetogénica se considera efectiva para perder peso, mejora ciertos parámetros bioquímicos, pero cuando se controlan las calorías, los resultados de la pérdida de peso tienden a ser similares a los de una dieta de control, aunque existen diferencias en la bioquímica sérica.

Palabras clave: Bajo en carbohidratos; Cetogénico; Perdida de peso.

\section{Introdução}

A transição nutricional apresenta um quadro atual de crescente excesso de peso na população. A gravidade desse processo gera uma mobilização mundial a fim de alertar a sociedade sobre as consequências da obesidade. No ano de 2016, mais de 1,9 bilhão de adultos estavam com excesso de peso e destes mais de 650 milhões eram obesos (WHO, 2017). No cenário nacional, a prevalência da obesidade passou de 11,8\% em 2006 para 20,3\% em 2019 (Brasil, 2019).

A obesidade é tratada como problema de saúde pública haja vista a interligação a várias patologias associadas, a exemplo da dislipidemia, diabetes, hipertensão, esteatose hepática. O indivíduo obeso tem maior predisposição a eventos cardiovasculares em comparação com indivíduos eutróficos (Carneiro, et al., 2003). A Diabetes Mellitus tem uma grande relação com a obesidade e a diminuição do excesso de peso é uma forma de tratamento eficaz (Faustino, et al., 2011). Existe um reconhecimento dessa enfermidade como uma epidemia mundial por ser de origem multifatorial (Enes \& Slater, 2010; WHO, 2020).

O dano metabólico em decorrência do alto grau de adiposidade é passível de monitoramento através de exames bioquímicos. Determinados marcadores são comprometidos. A resistência à insulina é uma das consequências desse agravo à saúde (Pereira et al., 2003; Pena et al., 2020). Diante desse cenário, estratégias para controle de peso corporal são aprimoradas com o objetivo de alcançar um emagrecimento saudável e melhoria dos parâmetros sanguíneos, para a reversão dos fatores de risco associados.

No que concerne ao tratamento dietético, constitui-se, a priori, na reeducação alimentar associada à adoção de hábitos saudáveis como a prática de exercícios físicos. De modo geral, as diretrizes da ABESO recomendam promoção de um balanço energético negativo com criação de um déficit calórico de 500 a 1000 quilocalorias, no qual se busca diminuição de peso corporal em torno de 0,5 a $1 \mathrm{~kg}$ por semana. Em relação ao corte de calorias, a maneira prática seria a redução da quantidade de gordura da dieta. Tendo em vista que as dietas balanceadas se caracterizam por serem compostas de $20 \%$ a $30 \%$ de gorduras, $55 \%$ a $60 \%$ de carboidratos e $15 \%$ a $20 \%$ de proteínas (ABESO, 2016). Essas porcentagens de macronutrientes são baseadas na RDA 
(ingestão dietética recomendada), que representa o valor de ingestão alimentar diária suficiente para atender a necessidade da maioria da população (Marchioni et al., 2004; DRIs, 2019). A restrição no teor de gordura se caracteriza como uma Dieta Baixa em Lipídios (DBL).

Existem divergências quanto às proporções de macronutrientes a serem utilizadas no planejamento dietético. Em relação a esse assunto, as Dietas Baixas em Carboidratos (DBC) detêm grande notoriedade a nível mundial e vêm sendo objeto de estudo há vários anos. Porém, ainda são geradas muitas dúvidas e controvérsias a respeito da mesma. A ABESO classifica essa estratégia dietética como "dieta da moda", e também questiona a segurança, a adequação nutricional e os resultados a longo prazo.

Uma dieta pobre em hidratos de carbono tem por definição uma composição menor que $45 \%$ desse macronutriente no Valor Calórico Total (VCT). Existe ainda uma subdivisão nas DBC, na qual uma dieta com $\leq 40 \%$ de HC representa uma restrição moderada e aquelas com menos de $10 \%$ são consideradas dietas muito restritas em carboidrato ou também chamadas de cetogênicas (Hashimoto et al, 2016). Desse modo, uma DBC apresenta uma margem extensa em sua definição, pois tem como parâmetro a recomendação da DRIs.

A justificativa dos autores para adoção dessa estratégia ao invés da distribuição habitual de macronutrientes seria, principalmente, pelo maior impacto hormonal no nível de insulina sérica, tendo em vista que o carboidrato é o principal estímulo insulínico dentre os macronutrientes. Sendo assim, uma menor ingestão desse macronutriente causa uma redução nos níveis sanguíneos de insulina, consequentemente possibilita uma maior ação de hormônios lipolíticos. Além disso, advoga-se sobre outros benefícios associados como maior perda de peso, controle do apetite pelo potencial sacietogênico, melhora da composição corporal e de parâmetros bioquímicos (Noakes \& Windt, 2017).

Uma dieta cetogênica é essencialmente caracterizada por forçar o corpo a utilizar os estoques de gordura como combustível através da restrição de carboidratos, consequentemente redução da glicemia, insulina sérica e estoque de glicogênio. Essa mobilização no tecido adiposo por meio da atividade da lipase hormônio sensível resulta em um aumento da formação de Acetil-CoA, que será oxidado a $\mathrm{CO}_{2}$ no ciclo de Krebs. A restrição glicídica provoca uma abundância de ácidos graxos oxidados, por esse motivo causa um grande volume de Acetil-CoA, que é o produto a ser oxidado no ciclo. Todavia, essa quantidade de Acetil-CoA vai além da capacidade do ciclo de ácido cítrico, por conseguinte, esse excesso de Acetil-CoA se acumula nos hepatócitos e é convertido em corpos cetônicos, isso elucida a nomenclatura dessa estratégia (Pogozelski et al., 2005).

Alfred Pennington foi um médico que propôs uma dieta rica em lipídios e proteínas, mas restrita em carboidrato para tratamento de obesos na década de 1950. A justificativa de Pennington para adoção desse tipo de dieta era que a obesidade não estava somente relacionada à hiperfagia, mas também a uma incapacidade do organismo de metabolizar adequadamente a glicose. Outro fator usado como justificativa é a capacidade sacietogênica da dieta, tendo em vista a ingestão elevada de gordura e proteínas. (Leith, 1961)

Ao contrário dos aminoácidos e ácidos graxos, o organismo é capaz de produzir glicose através de substratos nãoglicídicos (McDonald, 1998). No entanto, culturalmente, a base da alimentação da população é composta de carboidratos, seguindo os preceitos da pirâmide alimentar (Philippi, 2015). Por esse motivo, existe uma resistência natural à adesão das DBC, principalmente quando se trata de restrição severa (dieta cetogênica). Todavia, embora toda dieta cetogênica seja uma DBC, nem toda DBC é cetogênica (Wilson \& Lowery, 2017). Desse modo, uma quantidade maior de hidratos de carbono pode influenciar o estado de cetose e reduzir os mecanismos envolvidos com a elevação dos corpos cetônicos.

O objetivo geral do estudo foi analisar a eficiência de uma dieta cetogênica na redução do peso corporal, além de descrever os resultados ponderais dessa dieta, apontar as alterações nos parâmetros bioquímicos, descrever seus efeitos colaterais e identificar possíveis efeitos na performance de praticantes de atividade física com a dieta cetogênica. 
A Dieta Cetogênica (DC) está consolidada na literatura como tratamento de crianças com epilepsia e vem sendo amplamente estudada como uso terapêutico em outras patologias, a exemplo de diabetes, câncer, depressão, esquizofrenia (Paoli et al, 2013; Wilson et al, 2017; Weber et al, 2020). Todavia, encontra-se sob questionamento quando se refere à estratégia para perda de peso e por esse motivo é objeto de estudo há muitos anos, na tentativa de elucidar mecanismos da dieta, assim como segurança e eficácia em longo prazo.

A fisiologia dessa dieta tem similaridades com o estado de jejum, não coincidentemente, pois a origem da dieta cetogênica está relacionada a esse estado, tendo em vista que a DC mimetiza o jejum, ou seja, metabolicamente os processos são semelhantes mesmo sem a restrição total de alimento.

\section{Metodologia}

Este trabalho se trata de uma revisão bibliográfica narrativa, realizada a partir de artigos científicos completos publicados nos últimos quatorze anos, disponíveis em portais PubMed (www.pubmed.gov), Scielo (www.scielo.org), Google Acadêmico (http://scholar.google.com.br), BioMed (www.ncbi.nlm.nih.gov), acessados por intermédio da Biblioteca Virtual em Saúde (BVS), utilizando os seguintes descritores: dieta cetogênica e perda de peso, ketogenic diet and weight loss, low carb diet, very low carb diet, Dieta com Restrição de Carboidratos, Dieta Hiperlipídica.

A pesquisa foi direcionada aos trabalhos em humanos submetidos a uma dieta restrita em carboidrato com relação à perda de peso e parâmetros bioquímicos. Portanto, foram excluídos os estudos em animais e os protocolos terapêuticos para epilepsia, diabetes, câncer e outras doenças. Dentre os artigos visualizados, foram escolhidos os que se enquadram no conceito de dieta cetogênica, ou seja, um consumo menor ou igual a $10 \%$ do valor calórico total ou 30 a $50 \mathrm{~g}$ de carboidrato por dia. Além disso, a seleção se baseou na clareza dos dados metodológicos relacionados à quantidade de carboidrato, tempo de intervenção e controle calórico. O processo de seleção dos artigos pode ser conferido na Figura 1.

Figura 1: Fluxograma de seleção dos artigos.

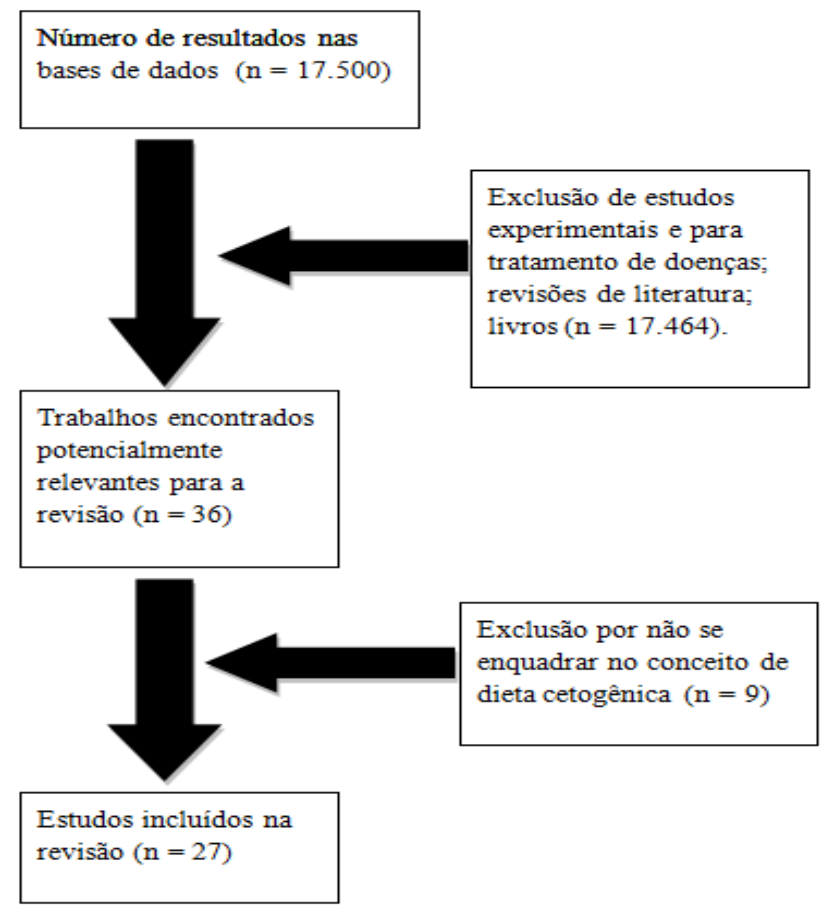

Fonte: Autores. 


\section{Resultados e Discussão}

Dentre os 27 artigos selecionados, 24 apresentaram grupo controle e em 17 trabalhos não houve restrição calórica. Em 11 artigos houve perda de peso significativa no grupo que seguiu uma dieta baixa em carboidrato em comparação ao controle. Desses onze, as calorias foram controladas em apenas 4 artigos, no restante o consumo foi ad libitum. Dentre os artigos selecionados, 13 estudos apresentaram perda de peso similar ao grupo controle. Nota-se nesses treze artigos que em 8 (oito) deles o consumo foi ad libitum, enquanto em 5 (cinco) houve controle de calorias (Figura 1).

Figura 1: Dieta com baixo carboidrato e a perda de peso com ou sem restrição de calorias.

\begin{tabular}{c|c|c|c|c}
\hline \multirow{2}{*}{ PROTOCOLOS } & \multicolumn{2}{|c|}{ Resultados de perda de peso } & \multirow{2}{*}{$\begin{array}{c}\text { Sem grupo } \\
\text { controle }\end{array}$} & \multirow{2}{*}{ TOTAL } \\
\cline { 2 - 5 } & Superiores & Similares & 2 & 17 \\
\cline { 2 - 5 } DC sem restrição & 7 & 8 & 1 & 10 \\
\hline DC com restrição & 4 & 5 & $\mathbf{3}$ & $\mathbf{2 7}$ \\
\hline Total & $\mathbf{1 1}$ & $\mathbf{1 3}$ & & \\
\hline
\end{tabular}

Fonte: Autores. 
Quadro 1 - Sequência de artigos sobre dietas com baixo carboidrato segundo autor, quantidade de carboidratos, duração do estudo e magnitude de resultados.

\begin{tabular}{|c|c|c|c|c|c|}
\hline $\mathbf{N}$ & AUTOR & Quantidade de carboidrato & $\begin{array}{c}\text { Tempo de } \\
\text { estudo }\end{array}$ & $\begin{array}{l}\text { Perda de peso em } \\
\text { relação ao grupo } \\
\text { controle }\end{array}$ & $\begin{array}{l}\text { Controle de } \\
\text { calorias }\end{array}$ \\
\hline 1 & Gregory et al, 2016 & 50 gramas & 6 semanas & $\uparrow$ & Não \\
\hline 2 & Bazzano et al, 2014 & $\begin{array}{l}40 \text { gramas (recomendação) } \\
127 \text { gramas (consumo ao final da } \\
\text { intervenção) }\end{array}$ & 12 meses & $\uparrow$ & Não \\
\hline 3 & Brinkworth et al, 2009 & $\begin{array}{c}20 \text { gramas na } 1^{\mathrm{a}} \text { fase } 40 \text { gramas na } \\
2^{\mathrm{a}} \text { fase }\end{array}$ & 8 semanas & $=$ & Sim \\
\hline 4 & Brinkworth et al, 2009 & $4 \%$ & 12 meses & $\uparrow$ & Sim \\
\hline 5 & Brinkworth et al, 2010 & $4 \%$ & 12 meses & $=$ & Sim \\
\hline 6 & Brinkworth et al, 2016 & $\begin{array}{c}20 \text { gramas na } 1^{\mathrm{a}} \text { fase } 40 \text { gramas na } \\
2^{\mathrm{a}} \text { fase }\end{array}$ & 12 meses & $=$ & Sim \\
\hline 7 & Gardner et al, 2007 & $\begin{array}{l}20 \text { gramas na } 1^{\mathrm{a}} \text { fase, } 50 \text { gramas na } \\
2^{\mathrm{a}} \text { fase }\end{array}$ & 12 meses & $\uparrow$ & Não \\
\hline 8 & Ruth et al, 2013 & 40 gramas & 12 semanas & $=$ & Sim \\
\hline 9 & Foster et al, 2010 & $\begin{array}{c}20 \text { gramas (até } 3 \text { meses). Com } \\
\text { acréscimo de } 5 \text { gramas por semana } \\
\text { depois dos } 3 \text { meses. }\end{array}$ & 24 meses & $=$ & Não \\
\hline 10 & Paoli et al, 2012 & 22 gramas & 30 dias & $=$ & Não \\
\hline 11 & Liu et al, 2013 & $\begin{array}{c}20 \text { gramas na } 1^{\mathrm{a}} \text { semana, aumento } \\
\text { semanal de } 10 \text { gramas até atingir } \\
120 \text { gramas }\end{array}$ & 12 semanas & $=$ & Não \\
\hline 12 & Partsalaki et al, 2012 & 40 gramas & 6 meses & $\uparrow$ & Não \\
\hline 13 & Yancy et al, 2010 & $\begin{array}{l}20 \text { gramas inicialmente, podendo } \\
\text { aumentar em } 5 \text { gramas por semana. } \\
\text { O consumo final foi de } 62 \text { gramas. }\end{array}$ & 48 semanas & $=$ & Não \\
\hline 14 & Krebs et al, 2010 & 20 gramas & 36 semanas & $=$ & Não \\
\hline 15 & Jabekk et al, 2010 & $\begin{array}{l}20 \text { gramas inicialmente, podendo } \\
\text { aumentar conforme julgasse } \\
\text { necessário. Consumo: } 23 \text { gramas }\end{array}$ & 10 semanas & $\uparrow$ & Não \\
\hline 16 & Rhyu et al, 2014 & 22 gramas & 3 semanas & $=$ & Sim \\
\hline 17 & Hu et al, 2016 & 40 gramas & 12 meses & $\uparrow$ & Não \\
\hline 18 & Urbain et al, 2017 & 20-40 gramas & 6 semanas & $*$ & Não \\
\hline 19 & Zinn et al, 2017 & 50 gramas & 10 semanas & $*$ & Não \\
\hline 20 & Johnstone et al, 2008 & $\begin{array}{c}4 \% \\
\begin{array}{c}\text { Porém o teor proteico foi igual para } \\
\text { os dois grupos }\end{array} \\
\end{array}$ & 4 semanas & $\uparrow$ & Não \\
\hline 21 & Johnstone et al, 2011 & $\begin{array}{c}4 \% \\
\begin{array}{c}\text { Teor proteico também foi igual em } \\
\text { ambas as dietas }\end{array} \\
\end{array}$ & 4 semanas & $\uparrow$ & Sim \\
\hline 22 & Kirk et al, 2012 & $\begin{array}{l}20 \text { gramas inicialmente. Depois } \\
\text { acrescentaria } 5 \text { a } 10 \text { gramas até } \\
\text { atingir o limite de } 60 \text { gramas. }\end{array}$ & 12 meses & $=$ & Não \\
\hline 23 & Halyburton et al, 2007 & $4 \%$ & 8 semanas & $\uparrow$ & Sim \\
\hline 24 & Hernandez et al, 2010 & 20 gramas & 6 semanas & $=$ & Não \\
\hline 25 & Vargas et al, 2018 & 42 gramas & 8 semanas & $=$ & Sim \\
\hline 26 & Mohorko et al, 2019 & $5-10 \%$ & 12 semanas & $*$ & Parcial \\
\hline 27 & Hall et al, 2021 & $10 \%$ & 2 semanas & $=$ & Não \\
\hline
\end{tabular}

Legenda: $(\uparrow)$ perda de peso superior ao grupo controle; $(=)$ perda de peso similar ao grupo controle; $(*)$ sem grupo controle. Fonte: Autores.

Gregory et al, (2016), submeteram 12 praticantes de CrossFit sem comorbidades a uma dieta cetogênica ad libitum, com duração de 6 semanas. De acordo com a média de consumo semanal, a ingestão de macronutrientes no grupo controle foi de $187.19 \pm 68.01 \mathrm{~g}$ de carboidrato, $73.47 \pm 18.86 \mathrm{~g}$ de lipídios e $80.45 \pm 18.61 \mathrm{~g}$ de proteína. Enquanto o grupo de dieta cetogênica foi de $44.42 \pm 16.46 \mathrm{~g}$ de carboidrato, $114.54 \pm 25.23 \mathrm{~g}$ de lipídios e $91.52 \pm 17.34 \mathrm{~g}$ de proteína. Em relação às calorias: 1745.73 $\pm 485.45 \mathrm{kcal}$ no grupo controle e $1580.66 \pm 283.37 \mathrm{kcal}$ no grupo cetogênico. Embora a única restrição na dieta cetogênica tenha sido a quantidade de carboidrato a ser ingerida, os participantes consumiram menor quantidade de quilocalorias 
comparados àqueles que seguiram a dieta controle. Como resultado, foi observado que a DC apresentou uma significativa perda de peso corporal, massa gorda, IMC e percentual de gordura em comparação com o grupo controle. Não houve diferença significativa na massa magra entre os grupos e o tempo total de desempenho diminuiu significativamente em ambos os grupos.

Os participantes da pesquisa tinham pelo menos um mês de experiência em CrossFit, que é um treinamento de alta intensidade, mesclando exercícios de ginástica, força, levantamento de peso olímpico, corrida, pliometria e movimentos funcionais. $\mathrm{O}$ grupo cetogênico, mesmo com a restrição severa de carboidrato, o que induz uma depleção de glicogênio, foi capaz de treinar ao menos quatro vezes por semana sem relatar efeito adverso. Além disso, houve melhora no teste de desempenho. Esse dado contrapõe Zajac et al, 2014, cuja conclusão foi de que em alta intensidade, a dieta cetogênica diminui a capacidade de trabalho devido aos estoques reduzidos de glicogênio.

Bazzano et al, (2014), durante 12 meses, conduziram um estudo nos quais homens e mulheres sem doença cardiovascular ou diabetes foram submetidos a uma dieta com restrição de carboidrato em 40g ou uma dieta com 30\% lipídios e 55\% carboidratos. Apesar da restrição recomendada, a ingestão glicídica pelos participantes ao final da intervenção ficou em torno de $127 \mathrm{~g}$ de carboidrato ao dia. Apesar de não haver meta calórica, o grupo em DBC consumiu uma quantidade de quilocalorias levemente inferior em comparação ao grupo DBL. Além disso, a porcentagem de proteína foi maior na dieta pobre em carboidrato. Os resultados mostraram que houve uma superioridade da DBC na perda de peso, aumento dos níveis de HDLc, redução da proporção colesterol total: HDL-c, redução do PCR e diminuição do risco para doença cardiovascular segundo o escore de risco de Framingham. Sem diferenças significativas entre os grupos nas reduções das concentrações de triglicerídeos e redução da circunferência de cintura. A quantidade de participantes que relataram efeitos adversos, como sintomas de constipação, fadiga, sede, poliúria, diarreia, azia, gases, náuseas, vômitos, alterações de apetite ou dor de cabeça não diferiu significativamente entre os grupos.

Gardner et al, (2007), realizaram uma comparação entre quatro tipos de dietas num espectro de restrição de carboidrato até uma ingestão glicídica elevada, no qual o objetivo principal foi analisar as alterações na perda de peso e fatores metabólicos em mulheres pré-menopáusicas no período de 12 meses. A meta para o grupo Atkins eram $20 \mathrm{~g}$ de carboidrato por dia que corresponde à fase de indução e depois até $50 \mathrm{~g}$ correspondente à segunda fase desta dieta. O grupo que seguiu a dieta da Zona, teve como meta de macronutriente $40 \%$ carboidrato, 30\% proteína e 30\% lipídios. O grupo LEARN (Lifestyle, Exercise, Attitudes, Relationships, and Nutrition) seguiu as diretrizes nutricionais convencionais com ingestão de $55 \%$ a $60 \%$ de carboidrato. Por último, o grupo da dieta Ornish tinha como meta restringir a ingestão de gordura em 10\% do valor calórico total. De acordo com as diretrizes apresentadas em cada livro, nos grupos Zona e LEARN houve meta específica de restrição de calorias, em contrapartida, nos grupos Atkins e Ornish não houve. Aos 12 meses, o grupo que seguiu a dieta Atkins perdeu maior quantidade de peso corporal em comparação com as outras dietas. Além disso, apresentou mudanças significativamente mais favoráveis nos níveis de HDL-c, triglicerídeos e pressão arterial.

Hall et al., (2021), compararam os efeitos de uma dieta cetogênica com uma dieta baixa em lipídios e à base de plantas em 21 adultos eutróficos de ambos os sexos. O grupo da dieta cetogênica consumiu 10\% de carboidratos e aproximadamente $75 \%$ de lipídios e $15 \%$ de proteína, além de consumirem ingredientes de origem animal. Já o grupo com dieta baixa em gorduras, 75\% de carboidratos, $10 \%$ de lipídios e a mesma quantidade de proteínas que o DC, ainda, excluiu qualquer ingrediente de origem animal, sendo completamente à base de plantas. Ambos os grupos restringiram alimentos ultraprocessados e apresentaram o consumo ad libitum durante duas semanas. O peso corporal reduziu nos dois grupos sem diferença significativa, porém, para o grupo DC a diminuição foi mais rápida durante a primeira semana, enquanto o grupo baixo em lipídios se manteve constante. Em relação à massa livre de gordura, o grupo DC apresentou maior perda principalmente durante a primeira semana, mas sem diferença significativa. Ao avaliar a massa gorda, o grupo DC iniciou uma maior perda após a primeira semana, ainda assim, não 
apresentou mudança significativa. Por outro lado, a dieta a base de plantas e baixa em gordura apresentou significativa e constante redução da massa gorda durante a intervenção.

Partsalaki et al, (2012), avaliaram o impacto metabólico de uma dieta cetogênica comparada com uma dieta hipocalórica em crianças e adolescentes obesos no período de seis meses. O grupo da DC ad libitum visou ingerir $20 \mathrm{~g}$ de carboidrato com um aumento gradual até atingir 30 a 40g por dia, desde que os participantes permanecessem em cetose. O grupo da dieta hipocalórica teve como recomendação a composição de 28 a 33\% lipídios e 50 a 55\% carboidrato juntamente a uma redução calórica de 500 quilocalorias. Os resultados encontrados após seis meses de intervenção foi que a DC se mostrou superior à dieta hipocalórica no que refere à perda de peso, perfil lipídico, níveis de adiponectina e sensibilidade à insulina. Por meio desses dados, o autor conclui que essa estratégia dietética pode ser utilizada no tratamento de crianças e adolescentes obesos.

Esse público também foi estudado por Kirk et al, (2012), que compararam a segurança e eficácia de duas dietas com modificações no carboidrato em comparação com dieta padrão no tratamento de crianças obesas. As crianças foram submetidas a uma intervenção de 3 meses em uma DBC ou RGL (dieta com Redução da Carga Glicêmica) ou dieta padrão. O grupo DBC realizou uma fase de restrição de carboidratos limitada a $20 \mathrm{~g} /$ dia. Após essa fase, pode-se acrescentar 5 a $10 \mathrm{~g}$ por semana até atingir o limite máximo de $60 \mathrm{~g} / \mathrm{dia}$, sem restrição energética. O grupo RGL foi orientado a limitar a ingestão de alimentos de alto Índice Glicêmico (IG), sem restrição aos alimentos de baixo IG e uma recomendação de ingestão de menor frequência dos alimentos com moderado IG, também sem restrição de calorias. O grupo da dieta padrão tinha como recomendação 55-60\% carboidrato, 10-15\% proteína e 30\% gordura. Com déficit calórico de 500 quilocalorias. Todos os grupos foram instruídos a utilizarem suplementação de vitaminas e minerais, além de uma recomendação de líquidos

Como resultado, todos os grupos apresentaram reduções no IMC, percentual de gordura e circunferência de cintura, sem diferenças significativas entre as dietas. Ao final dos 12 meses, o grupo DBC obteve melhores resultados nos níveis de triglicerídeos e HDL-c, enquanto a dieta padrão melhorou glicemia em jejum, insulina e HDL-c. A dieta RGL melhorou insulina em jejum e LDL-c. No entanto, os participantes do grupo DBC tiveram a menor adesão dietética comparados aos outros grupos. Esse dado mostra que a dieta aplicada não foi muito aceitável, dessa maneira, o estudo levantou a hipótese de que uma versão menos restrita dessa estratégia possa resultar numa maior adesão mantendo os resultados favoráveis no tratamento da obesidade. Além disso, sugere que os resultados a longo prazo numa DBC estão ligados à adesão, no qual, apesar de não significativo estatisticamente, ocorreu um rebote na redução do percentual de gordura e IMC. Com isso, criase a possibilidade de que uma adesão constante na dieta poderia mostrar resultados diferentes entre os grupos.

Já Mohorko et al, (2019) avaliaram perda de peso, performance, função cognitiva, comportamento alimentar e perfil metabólico de adultos obesos durante 12 semanas de dieta cetogênica associada ao exercício físico. Os parâmetros foram avaliados na $1^{\mathrm{a}}, 2^{\mathrm{a}}, 4^{\mathrm{a}}, 8^{\mathrm{a}}$ e $12^{\mathrm{a}}$ semana. A intervenção consistiu na restrição calórica nas primeiras duas semanas com posterior ad libitum, porém com manutenção da restrição de carboidratos de no máximo 10\%. A distribuição dos demais macronutrientes foi de aproximadamente $20 \%$ proteína e $75 \%$ lipídios. Como resultado, os participantes relataram aumento significativo da sensação de plenitude. Além disso, houve redução do peso corporal, sendo maior nos participantes homens. Em relação à massa gorda, ocorreu redução significativa na primeira semana de intervenção nas mulheres, porém, após a $2^{\mathrm{a}}$ semana não foi significativa, diferente do que ocorreu com os homens, que mantiveram a perda progressiva de massa gorda até a última semana de intervenção. Na performance, houve melhoria considerável em ambos os sexos, o que o autor associa à perda de peso.

No estudo, ainda, a função cognitiva dos participantes, após 12 semanas de dieta cetogênica, obteve melhora em testes de memória e velocidade de processamento. Em relação ao comportamento, houve êxito na percepção de imagem corporal e redução da fome emocional nos indivíduos. Na avaliação dos exames bioquímicos a glicose sérica e insulina reduziram significativamente após a $1^{\mathrm{a}}$ semana, voltando à normalidade após a $4^{\mathrm{a}}$ semana. Os triglicerídeos reduziram progressivamente apenas nas mulheres e permaneceram inalterados nos homens até o fim da intervenção. Já no colesterol LDL houve redução logo 
após a primeira semana, em ambos os sexos. Nas mulheres, ainda, a proteína $\mathrm{C}$ reativa diminuiu com 1 semana de intervenção. Ainda, Mohorko et al, 2019 relatou alteração nos hormônios adiponectina e leptina, ambos reduziram após 8 e 2 semanas respectivamente em todos os participantes. Já o cortisol, aumentou após 8 semanas em ambos os sexos.

Para avaliar a adesão a uma DBC ou DBL em relação a perda de peso e fatores de risco cardiovascular, Hu et al, (2016), no período de 12 meses testaram uma DBC com limite 40 g de carboidrato e uma DBL com 30\% de lipídios e menos de 7\% de gordura saturada, sem meta específica de calorias nos dois grupos. Como resultado, apesar da adesão semelhante em ambos os grupos, a DBC foi associada a maiores reduções no peso e melhora da composição corporal. Em relação aos parâmetros metabólicos, não foi observada associação consistente entre os indicadores de adesão a uma DBC e alterações na pressão sanguínea, perfil lipídico, glicemia e PCR ao final da intervenção.

Para avaliar a adesão em longo prazo das DBC, Foster et al, (2010), submeteram 153 indivíduos a uma DBC com ingestão de $20 \mathrm{~g}$ de carboidrato nos três meses iniciais, nos meses seguintes foram instruídos a acrescentar semanalmente cinco g diárias até obter o peso desejado. Para o grupo de DBL ( $\leq 30 \%)$, foram randomizados 154 participantes para seguir uma dieta com ingestão calórica limitada em $1200 \mathrm{kcal}$ a 1800 kcal. Ambos os grupos receberam suporte comportamental com reuniões frequentes a fim de estabelecer uma consistência no planejamento. O nível de atividade seguiu um padrão para todos os participantes assim como a suplementação de vitaminas e minerais fornecida pelo estudo. A avaliação foi baseada nos registros alimentares, relatos nas reuniões e coleta de dados realizadas nos $3^{\circ}, 6^{\circ}, 12^{\circ}$ e $24^{\circ}$ mês.

Como resultado, após 24 meses, não houve diferença significativa entre os grupos na perda de peso corporal, também não houve diferenças na densidade mineral óssea e na composição corporal e concentrações de cetonas urinárias. O parâmetro que diferiu entre as dietas foram os níveis de HDL-c, o grupo DBC apresentou maiores elevações em comparação com a DBL. A perda de peso em ambos os grupos foi de aproximadamente $11 \%$ aos 6 e 12 meses, porém houve recuperação de massa corporal, consequentemente, o percentual de perda foi reduzido para 7\% aos 24 meses. Os sintomas adversos como halitose, perda de cabelo e boca seca foram limitados aos 6 meses de intervenção, exceto pela constipação intestinal. Os dados obtidos ao final da intervenção mostram uma diminuição da diferença entre os grupos nos parâmetros avaliados, no qual, provavelmente, devido ao aumento na ingestão de carboidrato no grupo DBC, as mudanças significativas nas concentrações de triglicerídeos, VLDL e pressão diastólica encontradas nos primeiros meses não persistiram nos meses subsequentes. Da mesma maneira, as reduções dos níveis de LDL-c no grupo DBL no $3^{\circ}$ e $6^{\circ}$ mês, não foram encontradas no $12^{\circ}$ e $24^{\circ}$ mês.

Johnstone et al, (2008), compararam os efeitos de uma dieta cetogênica hiperproteica e uma dieta moderada em carboidrato (DMC) com alto teor proteico na sensação de fome e perda de peso em 17 homens obesos no período de 4 semanas. Ambas foram ad libitum com a mesma ingestão proteica de 30\%. Na DC, os carboidratos se limitaram a 4\%, os lipídios em 66\%. Na DMC o limite foi de 35\% para hidratos de carbono e os lipídios em 35\%. Os resultados mostraram aumento da saciedade e perda de peso significativamente maior no grupo DC do que no grupo DMC. Esse mesmo autor conduziu um estudo com 16 indivíduos obesos, em dietas isoenergéticas com as mesmas características. Os resultados apontaram perdas semelhantes de massa gorda nos grupos, e perda de peso total maior no grupo DC, porém segundo o autor, essa perda total pode ter sido influenciada pela perda de água maior nesse grupo em relação aos participantes em DMC. Marcadores plasmáticos de resistência à insulina, lipemia e inflamação melhoraram de forma semelhante. Não houve alteração em outros marcadores cardiovasculares, sugerindo que ambas são seguras no período de 4 semanas e poderiam ser usadas para alcançar perda de peso considerável, melhorar mortalidade e morbidade em pacientes obesos (Johnstone et al, 2011).

Halyburton et al, (2007), compararam os efeitos de uma dieta com baixo teor de carboidrato e alto em gordura (LCHF) com uma dieta de alto teor de carboidrato e baixo em gordura (HCLF) sobre o humor e a função cognitiva. A LCHF teve como recomendação de macronutrientes $4 \%$ de carboidrato, $35 \%$ de proteína e $61 \%$ de lipídios (20\% gordura saturada), enquanto a HCLF foi $46 \%$ de carboidrato, $24 \%$ de proteína e $30 \%$ de lipídios ( $<8 \%$ gordura saturada). As dietas foram isocalóricas e com 
restrição energética moderada de aproximadamente $30 \%$ no período de oito semanas. Como resultado, o grupo em dieta LCHF apresentou uma perda de peso significativamente maior do que o grupo HCLF. Além disso, os participantes que consumiram uma dieta LCHF não apresentaram diferenças nas medidas de humor em comparação ao grupo HCLF. As medidas de função cognitiva mostraram melhora significativa em ambos os grupos. No entanto, a dieta LCHF resultou em uma velocidade de processamento inferior ao grupo HCLF.

Jabekk et al, (2010), testaram uma dieta cetogênica associada ao treinamento resistido em 16 mulheres sedentárias com sobrepeso no período de 10 semanas. A composição da DC foi $6 \%$ carboidrato, $66 \%$ lipídios e $22 \%$ proteína; a dieta do grupo controle teve a seguinte composição: $41 \%$ carboidrato, $34 \%$ lipídios e $17 \%$ proteínas. A DC limitou a ingestão de carboidrato em $20 \mathrm{~g}$ inicialmente e os participantes poderiam aumentar gradualmente conforme julgassem necessário, desde que a mudança de cor nas tiras urinárias de cetose permanecesse. Dessa maneira, não houve restrição energética neste grupo. Apesar disso, os participantes consumiram uma quantidade menor de calorias em comparação ao grupo controle. Os resultados mostraram que o grupo DC reduziu a massa gorda, sem alterar significativamente a massa magra, enquanto o grupo controle aumentou a massa magra sem alterar significativamente a massa gorda. O perfil lipídico e a glicemia não foram afetados de forma significativa pelas intervenções.

Paoli et al, (2012), utilizaram durante 30 dias uma DC ad libitum em 9 atletas de ginástica de elite com ingestão de 4,5\% de carboidrato (limite de 22 g), 40,7\% de proteína e 54,8\% de lipídios durante 30 dias, para analisar os efeitos sobre a composição corporal e desempenho atlético. Após 3 meses os mesmos atletas utilizaram uma dieta controle com 46,8\% carboidrato, 14,7\% de proteína e 38,5\% lipídios por 30 dias. Essa distância temporal permitiu uma análise comparativa dos resultados de ambas dietas. Como resultado, houve uma redução média de peso corporal, massa gorda, percentual de gordura e aumento da porcentagem de massa magra. No entanto, sem diferenças significativas entre os grupos nas variáveis antropométricas e teste de desempenho. Os dados reforçam a hipótese do autor na possibilidade de utilização da dieta cetogênica na preparação de atletas que necessitam de enquadramento em categorias de peso. De acordo com esse estudo, essa estratégia dietética seria viável por não comprometer o desempenho dos atletas enquanto melhora a composição corporal.

Liu et al, (2013), analisaram os efeitos de uma DBC contra uma DBL em 50 mulheres chinesas com sobrepeso/obesidade, para observar a adesão ao protocolo uma vez que a dieta ocidental habitual concentra um alto percentual de carboidrato, aproximadamente 57\%. A DBC teve como limite $20 \mathrm{~g}$ de carboidrato na primeira semana com aumento semanal de $10 \mathrm{~g}$ até atingir o limite de $120 \mathrm{~g}$ de HC. Os participantes que seguiram a DBC apenas limitaram a ingestão de carboidrato, sendo os demais macronutrientes ad libitum conforme o planejamento. Já o grupo na DBL teve restrição energética. Constatouse que ambos os grupos reduziram a ingestão, mas o grupo DBC consumiu uma quantidade menor de calorias. Além disso, não houve diferença na adesão à dieta dos grupos. Como resultado, não foi encontrada diferença significativa entre as dietas em relação a perda de peso, IMC, circunferência de cintura e quadril, pressão sanguínea, massa gorda e massa magra. A DBC foi mais favorável em parâmetros metabólicos, com aumento do HDL-c e redução na relação CT: HDL-c e TG: HDL-c, porém, obtiveram maiores níveis de uréia devido à maior ingestão proteica na dieta. No que se refere às enzimas hepáticas, não foram encontradas diferenças entre os grupos.

Yancy et al, (2010), acompanhando 146 obesos em um período de 48 semanas, compararam uma DBC sem restrição calórica com uma DBL com restrição energética de 500 a 1000 calorias e esta última adicionada de 120 mg de Orlistate (também conhecido como Xenical) antes das refeições três vezes ao dia para diminuir a absorção de gordura através de ação nas lipases. Houve um melhor resultado na pressão sanguínea no grupo DBC. Os níveis de triglicerídeos e HDL-c melhoraram de forma semelhante entre os grupos, assim como a perda de peso. A DBC obteve resultados similares a uma DBL com fármaco antiobesidade. Ambas as intervenções melhoraram fatores de risco cardiovascular, porém a DBC teve efeito mais favorável sobre a pressão sanguínea. Apesar da natureza ad libitum, o grupo DBC restringiu involuntariamente as calorias. Ao final da 
intervenção, a ingestão de carboidrato foi de $62 \mathrm{~g}$. Efeitos adversos foram relatados em ambos os protocolos, especialmente a nível de sistema gastrointestinal, no qual devido ao uso do fármaco Orlistate, os participantes do grupo DBL relataram mais efeitos adversos e no grupo DBC, a constipação foi o principal sintoma relatado pelos participantes.

Krebs et al, (2010), avaliaram a eficácia e segurança de uma dieta hiperproteica e restrita em carboidrato por 13 semanas comparada com uma DBL na perda de peso em adolescentes com obesidade grave. A DBC consistia na ingestão de $20 \mathrm{~g}$ de carboidrato e uma ingestão de proteínas em torno de 2,0 a 2,5 g/Kg de peso corporal ideal por dia, sem restrição energética neste grupo. Para a DBL foi instituída uma restrição calórica e com limite de 30\% de lipídios no consumo diário. Os dois grupos foram encorajados a realizar uma atividade física vigorosa de 30 minutos por dia. Além disso, foi fornecida suplementação de cálcio e vitaminas e minerais para ambos os grupos, com recomendação de ingestão hídrica superior a aproximadamente 1400 mililitros. Os resultados mostraram uma redução superior no IMC no grupo DBC durante o acompanhamento de 13 semanas. A superioridade persistiu até $24^{\mathrm{a}}$ semana, mas na $36^{\mathrm{a}}$ semana não houve diferença significativa entre os grupos.

Os dados da composição corporal mostraram que os participantes na DBC perderam mais gordura, porém, também perderam maior quantidade de massa magra, mesmo com uma ingestão elevada de proteína. Em relação aos parâmetros metabólicos, os dois grupos melhoraram significativamente o Colesterol Total e LDL-c. Quanto aos triglicerídeos e HDL-c, os resultados mais favoráveis foram no grupo DBC. Quanto aos efeitos adversos não houve diferença significativa entre os grupos, exceto pelo sintoma de dor de cabeça relatado por mais participantes do grupo DBC.

Hernandez et al, (2010), compararam o efeito de uma DBC com uma dieta de alto teor de CHO em variáveis metabólicas durante a perda de peso no período de seis semanas. Uma avaliação de 24 horas em parâmetros metabólicos foi realizada no início e no final da intervenção. Glicemia, insulina, ácidos graxos livres e triglicerídeos foram medidos a cada hora durante as refeições. Já o colesterol remanescente de lipoproteína foi medido a cada 4 horas. O grupo DBC apenas limitou a ingestão de carboidrato em $20 \mathrm{~g}$ sem restrição energética. Enquanto a dieta padrão teve uma recomendação de calorias, sendo composta de $30 \%$ lipídios, $15 \%$ proteína e $55 \%$ carboidrato. Os resultados mostraram que a perda de peso foi semelhante entre as dietas, mas apenas a dieta com alto teor de gordura aumentou as concentrações de LDL-c. Este efeito foi relacionado à falta de supressão de ácidos graxos livres em jejum e 24 horas.

Ruth et al, (2013), realizaram uma intervenção de doze semanas comparando uma DBC (40 g de carboidrato, 35\% proteína e $60 \%$ lipídios) com uma DBL (60\% carboidrato, 15\% proteína e $25 \%$ lipídios) sobre o peso corporal, fatores de risco cardiovascular e marcadores inflamatórios em indivíduos obesos. Os sujeitos foram submetidos a um déficit calórico de $500 \mathrm{kcal}$ com o objetivo de induzir perda de 5 a $10 \%$ do peso total ao longo das doze semanas. O grupo DBC apresentou maiores reduções médias nos níveis de triglicerídeos e Proteína C-Reativa, além de maiores elevações nas concentrações de HDL-c e adiponectina total quando comparado com o DBL. Não houve diferenças nos níveis de adiponectina do tecido adiposo e TNF- $\alpha$ após a intervenção das dietas. Observou-se diminuição similar do peso corporal entre os grupos, porém uma maior proporção (78\%) de indivíduos que seguiram a dieta DBC perderam mais de 5\% do peso em comparação com os que seguiram a dieta DBL (53\%). Todos os participantes da pesquisa melhoraram a proporção de massa magra e gordura corporal em relação ao início da intervenção.

Brinkworth et al, (2009), analisaram os efeitos de uma DBC e uma DBL no período de 12 meses. Ambas as dietas com restrição energética, a diferença se baseava na composição de macronutriente, no qual na DBC foi $4 \%$ carboidrato, 35\% proteína e $61 \%$ lipídios (20\% gordura saturada), já a DBL foi de $46 \%$ carboidrato, $24 \%$ proteína e $30 \%$ lipídios ( $<8 \%$ gordura saturada). A DBC tinha um limite de $20 \mathrm{~g}$ nas primeiras 8 semanas, após esse período o limite foi aumentado para $40 \mathrm{~g}$ de carboidrato. A fim de manter as dietas em situação isocalórica, na DBL também houve um acréscimo de calorias para equivaler energeticamente o protocolo da DBC. Não houve diferença estatisticamente significativa na perda de peso corporal ao compararem os grupos. O consumo total de energia foi semelhante entre as dietas, assim como os níveis de atividade física. A análise global mostrou uma 
diminuição nos dois grupos no que se refere à massa gorda, massa magra e gordura abdominal, ou seja, não houve influência da composição de macronutrientes nesses parâmetros. Quanto ao perfil lipídico, a DBC resultou em maiores elevações nos níveis de colesterol total, LDL-c, HDL-c e reduções nos triglicerídeos séricos do que na DBL. Ambas as dietas mostraram melhorias significativas na pressão sanguínea, glicemia, resistência à insulina e PCR.

No mesmo ano, o autor analisou os efeitos das dietas na capacidade aeróbia, força muscular e adaptações metabólicas do exercício em indivíduos obesos sedentários no período de 8 semanas. Em relação ao desempenho, não foram encontradas diferenças na DBC e DBL quanto aos efeitos sobre o exercício, percepção de fadiga e esforço. Percebeu-se que na DBC ocorre um aumento da oxidação de gordura durante o exercício aeróbio submáximo. Dessa forma, o autor conclui que a restrição de carboidrato não prejudicou a função física e a tolerância ao exercício. (Brinkworth et al., 2009).

Quanto à função renal, esse mesmo autor observou que não houve aumento nos níveis de creatinina sérica e taxa de filtração glomerular. Dessa maneira, o autor concluiu que a função renal de homens e mulheres com obesidade abdominal não foi afetada negativamente pela DBC em um período de 1 ano. (Brinkworth et al., 2010)

Em 2016 Brinkworth et al, submeteram indivíduos obesos aos mesmos protocolos de dietas por 8 semanas de intervenção. Os resultados apontaram que não houve diferenças entre os grupos nas medidas avaliadas após 1 ano de intervenção. A perda de peso média foi de aproximadamente $13,6 \pm 8,1 \%$, sem afetar os marcadores de saúde óssea.

Rhyu et al, (2014), investigaram o efeito de uma dieta cetogênica na composição corporal, desempenho físico e citocinas inflamatórias em 20 atletas de taekwondo no período de 3 semanas. A DC teve como o limite de ingestão $22 \mathrm{~g}$ de carboidrato, gerando a seguinte composição: 4,3\%, 40,7\% e 55\% para carboidrato, proteína e lipídios respectivamente. Já o grupo controle tinha uma composição semelhante aos preceitos da Dieta da Zona, $40 \%$ carboidrato 30\% proteína e $30 \%$ lipídios; essa proporção é a recomendação da Sociedade Coreana de Nutrição. Ambas as dietas foram submetidas a uma restrição energética. Como resultado, não houve diferença entre os grupos na perda de peso corporal, percentual de gordura, IMC e massa magra. Apesar disso, o grupo DC melhorou a capacidade aeróbia e resistência à fadiga, além de ter atenuado a resposta inflamatória à perda de peso, por mostrar menores elevações no TNF- $\alpha$.

Urbain et al, (2017), investigaram o impacto de uma dieta cetogênica ad libitum na composição corporal, desempenho físico e parâmetros bioquímicos em indivíduos saudáveis. A DC era limitada em 20 a $40 \mathrm{~g}$ de carboidrato, com a seguinte proporção: $75 \%$ lipídios, $15-20 \%$ proteína e 5-10\% carboidrato. A investigação se baseou na análise do período anterior e posterior à intervenção de 6 semanas em 42 indivíduos. O resultado mostrou uma perda de peso igual em relação a massa gorda e massa magra, além disso houve um impacto ligeiramente negativo no desempenho físico. No que se referem aos parâmetros bioquímicos, as concentrações de triglicerídeos e HDL-c permaneceram inalteradas, enquanto os níveis de LDL-C e CT sofreram elevações. Houve diminuição na glicemia, insulina e IGF-1. O autor conclui que a DC pode ser motivo de preocupação para atletas competitivos.

O estudo de Zinn et al, (2017), corrobora com essa conclusão pois ao avaliar os efeitos de uma dieta cetogênica na composição corporal e desempenho físico em 5 atletas de endurance no período de 10 semanas obteve como resultado um desempenho aeróbio máximo afetado negativamente, apesar da redução da gordura corporal. A DC era limitada em 50g de carboidrato, 1,5g de proteína/Kg de peso corporal e lipídios ad libitum. Nos dois estudos supracitados, não houve grupo controle com dieta padrão na intervenção, o controle foi análise de antes e depois da DC nos mesmos indivíduos.

Vargas et al, (2018), avaliaram a eficácia de uma intervenção de 8 semanas de dieta cetogênica em homens treinados durante treino de resistência. Os participantes foram divididos em grupo controle, dieta cetogênica e não-cetogênica ambas hiper energéticas (39 kcal $/ \mathrm{kg}$ ). $\mathrm{O}$ grupo dieta não-cetogênica apresentou uma distribuição dos macronutrientes de: 55\% de carboidrato, $20 \%$ proteína e $25 \%$ lipídio. Já o grupo da dieta cetogênica, 10\% de carboidrato, 20\% proteína e $70 \%$ lipídio. Durante as 8 semanas os indivíduos seguiram o protocolo de treino resistido 4 vezes por semana. Como resultado, o grupo dieta cetogênica 
apresentou redução na massa gorda e tecido adiposo visceral, porém com diminuição não significativa da massa livre de gordura e do peso corporal quando comparado ao grupo não-cetogênico.

Ao analisar os resultados, nota-se que a DC causou perda de peso em todos os artigos levantados, portanto, foi eficiente nesse parâmetro. No qual há estudo de no mínimo 2 semanas e no máximo 24 meses, o que possibilitou avaliar a resposta em longo prazo. Apesar da perda de peso em comum, não houve unanimidade entre as conclusões dos autores em relação a dieta cetogênica ser totalmente favorável. As principais alegações estão relacionadas a decréscimos na performance, dificuldade de adesão e preocupações quanto a efeitos colaterais.

Em alguns artigos, a ingestão de carboidrato aumentou consideravelmente em comparação ao consumo inicial, porém, estavam de acordo com o protocolo da dieta Atkins conforme metodologia adotada pelos estudos. Quanto ao consumo de carboidrato, a menor ingestão foi de $4 \%$ do VCT e a maior ficou em torno de $35 \%$ ao final de um dos estudos selecionados. Porém no estudo de Foster et al, 2010, não foi relatado o consumo ao final dos 24 meses, o que poderia mostrar uma ingestão possivelmente superior, pois foi o estudo de maior duração utilizando o conceito da dieta Atkins.

As principais limitações em alguns dos artigos levantados foram curto período de intervenção e pequeno tamanho de amostra. Em dois artigos dos estudos levantados, têm como limitação a ausência de grupo controle, o que impossibilitou a comparação entre dietas no tocante à perda de peso e performance. Em Yancy et al, 2010, destaca-se o subsídio fornecido pela Fundação Robert C. Atkins aos pesquisadores Yancy e Westman. Esse dado está explícito na publicação. Essa pesquisa era de interesse da Fundação, tendo em vista que o protocolo utilizado no estudo para a DBC está em conformidade com as diretrizes da dieta Atkins. Obviamente, essa informação se restringe apenas a uma suposição de uma possível influência no estudo.

Os resultados encontrados através dos artigos selecionados mostram uma maior quantidade de estudos que apresentam similaridades nos resultados da perda de peso corporal em comparação ao grupo controle. Em relação aos parâmetros bioquímicos, existe uma forte tendência a aumento nos níveis de HDL-c, diminuição da pressão sanguínea e redução dos triglicerídeos nos grupos com dieta cetogênica. Isso mostra que mesmo em condições isocalóricas, diferenças na composição de macronutrientes mostram resultados divergentes quanto a alguns parâmetros bioquímicos.

Os efeitos adversos documentados de uma DC para tratamento da obesidade se restringem, geralmente, a sintomas relacionados ao período de adaptação como dor de cabeça, letargia, constipação, pois artigos avaliando função renal, hepática e óssea não mostraram prejuízos diferentemente dos artigos com DC em crianças epilépticas. Dessa forma, infere-se que os resultados divergentes encontrados nesses artigos não estejam diretamente associados a dieta cetogênica, o que pode ser influência dos fármacos antiepiléticos, fisiopatologia da doença e tratamento à qual a criança foi submetida. A exemplo da restrição de fluídos que objetiva indução mais rápida ao estado de cetose, porém pode favorecer o surgimento de cálculos renais.

Com isso, necessita-se de cautela na comparação de dados obtidos de artigos entre não-portadores e portadores de epilepsia. Além do mais, a composição de macronutrientes costuma apresentar diferenças, principalmente em uma maior ingestão de proteínas.

Embora a recomendação geral limite a ingestão de carboidrato em menos de $50 \mathrm{~g}$ para indução ao estado de cetose, existe uma individualidade nas elevações de corpos cetônicos quanto ao consumo total de hidratos de carbono (Wilson et al, 2017). Em razão disso, determinados protocolos permitem um aumento no limite de carboidrato desde que haja monitoramento das cetonas urinárias por meio de tiras de teste. No entanto, essas tiras para medir cetose não detectam a oxidação geral. Além disso, são limitadas em prever os níveis plasmáticos de cetonas, porque as concentrações urinárias se correlacionam mal com os níveis sanguíneos (Pogozelski, 2005).

O principal benefício da DC está relacionado a não necessidade de uma restrição calórica, tendo em vista que involuntariamente quem segue essa dieta consome uma quantidade menor de calorias devido à supressão do apetite pelos lipídios, proteínas e corpos cetônicos. Dessa forma, obtêm-se resultados similares ou até superiores a uma DBL hipocalórica quanto à 
perda de peso. Quando há controle de calorias, a perda de peso tende a ser similar entre a DC e DBL. Porém, há diferenças nos efeitos no perfil lipídico, pressão sanguínea e marcadores de inflamação em alguns estudos. Além disso, ocorre uma maior influência nas sensações de fome.

Em relação à segurança da DC, dentre os artigos levantados, nota-se uma tendência à elevação dos níveis de LDL-c em alguns casos, porém antes de correlacionar com aumento do risco de doença cardiovascular, necessita-se saber o tamanho da partícula dessa lipoproteína que sofreu elevação. Além disso, o aumento dos níveis de HDL-c é um indicador benéfico na redução de risco para DCV, no qual a elevação nas concentrações dessa lipoproteína através da DC mostra uma eficácia comparável ao tratamento farmacológico utilizado para aumento do HDL-c (Foster et al, 2010).

Outra preocupação está relacionada ao aumento na ingestão de gordura saturada na dieta, pois o valor consumido chega ao dobro da recomendação. Porém, em um estudo prospectivo de coorte em 18 países mostrou que a gordura total e os tipos de gordura não foram associados a doenças cardiovasculares, infarto do miocárdio ou mortalidade por doenças cardiovasculares, além disso a gordura saturada apresentou uma associação inversa com AVC (Dehghan et al, 2017).

Existe a necessidade de um acompanhamento nutricional frequente para aumentar a adesão do paciente ao plano alimentar e proporcionar um suporte para controle dos efeitos adversos. Dentre esses, o sintoma que comumente perdura além do período de cetoadaptação é a constipação. No estudo de Hall et al., 2021, o grupo DC consumiu menos que o dobro de fibra quando comparado ao grupo baixo em gordura e a base de plantas, o que pode explicar a tendência à constipação em dietas baixas em carboidratos. Em relação a isso, o combate se dá por meio do consumo de fibras através de vegetais e/ou suplementação Outro cuidado se atém às vitaminas e minerais para que o paciente não sofra deficiência de micronutrientes, devido à exclusão de alimentos fonte de carboidratos ricos em vitaminas. Em razão disso, o consumo de vegetais de baixo amido e frutas com baixa carga glicêmica são recomendados dentro do limite instituído para hidratos de carbono.

A dieta cetogênica é bastante utilizada como estratégia inicial dentro de um planejamento nutricional, no qual se inicia com uma restrição mais severa na ingestão de carboidrato para depois aumentá-la gradualmente. Porém, a DC também é utilizada por períodos mais prolongados com ou sem recargas de carboidrato. De todo modo, a conduta é dependente do paciente, pois existe quem se habitue facilmente a este tipo de dieta e segue de forma consistente como um estilo de vida. Entretanto, devido aos hábitos alimentares instituídos há bastante tempo, o consumo de carboidrato difere exorbitantemente de uma DC. Por esse motivo, uma restrição menos severa poderia evitar um impacto brusco que porventura possa induzir uma má adaptação. Dessa maneira, a escolha ainda seria por uma DBC, caso fosse a conduta escolhida, porém não necessariamente uma DC.

A utilização da dieta cetogênica em atletas ainda não apresenta um consenso e por esse motivo necessita de mais estudos com este público. Principalmente a influência no desempenho físico em diferentes modalidades, pois o objetivo é a melhora da composição corporal com concomitante manutenção ou, se possível, aumento da performance. Porém, existe a dificuldade no recrutamento de atletas de alta performance como amostra de uma pesquisa.

\section{Considerações Finais}

Através dos dados expostos, objetiva-se que a dieta cetogênica seja mais uma opção na conduta do nutricionista. No qual após a anamnese, o profissional de nutrição possa escolher dentre as estratégias dietéticas, a que melhor se enquadre na necessidade, estilo de vida e objetivo do paciente. Dessa maneira, a DC e as DBC em geral não estariam mais vinculadas a alcunha de "dieta da moda", em razão da base literária em anos de estudos. E assim, o nutricionista pode ter a liberdade de prescrever um plano alimentar seguro e eficiente mesmo que vá de encontro às recomendações nutricionais vigentes.

Em casos de indivíduos inaptos a seguir a DC talvez possa resultar em um estresse decorrente do desejo incontrolável por alimentos ricos em hidratos de carbono. Por esse motivo, precisa-se que o nutricionista analise a aptidão do paciente ao planejamento nutricional a ser adotado, com o intuito de que esteja em conformidade com as preferências alimentares relatadas. 
Pois, dessa maneira, evita-se um estresse desnecessário por causa de uma estratégia nutricional inadequada ao paciente.

Diante da relevância deste tema, são necessários novos estudos e revisões sobre a dieta cetogênica como uma estratégia para perda de peso, como também avaliar as aplicabilidades como terapia adjuvante no tratamento de autismo, síndrome do ovário policístico, diabetes, dor crônica, câncer, doenças neurológicas e outras comorbidades.

\section{Referências}

ABESO, Diretrizes (2016). https://abeso.org.br/diretrizes/

Atkins, R. C. (2002). Dr. Atkins'New Diet Revolution. Government Institutes.

Bazzano, L. A., Hu, T., Reynolds, K., Yao, L., Bunol, C., Liu, Y., \& He, J. (2014). Effects of Low-Carbohydrate and Low-Fat Diets. Annals of Internal Medicine, 161(5), 309 .

Brasil. (2020). Secretaria de Vigilância em Saúde. Vigitel Brasil 2019: vigilância de fatores de risco e proteção para doenças crônicas por inquérito telefônico : estimativas sobre frequência e distribuição sociodemográfica de fatores de risco e proteção para doenças crônicas nas capitais dos 26 estados brasileiros e no Distrito Federal em 2019. Secretaria de Vigilância em Saúde. - Brasília: Ministério da Saúde, 2020

Brinkworth, G. D., Noakes, M., Clifton, P. M., \& Buckley, J. D. (2009). Effects of a Low Carbohydrate Weight Loss Diet on Exercise Capacity and Tolerance in Obese Subjects. Obesity, 17(10), 1916-1923.

Brinkworth, G. D., Noakes, M., Buckley, J. D., Keogh, J. B., \& Clifton, P. M. (2009). Long-term effects of a very-low-carbohydrate weight loss diet compared with an isocaloric low-fat diet after 12 mo. The American Journal of Clinical Nutrition, 90(1), $23-32$.

Brinkworth, G. D., Wycherley, T. P., Noakes, M., Buckley, J. D., \& Clifton, P. M. (2016). Long-term effects of a very-low-carbohydrate weight-loss diet and an isocaloric low-fat diet on bone health in obese adults. Nutrition, 32(9), 1033-1036.

Brinkworth, G. D., Buckley, J. D., Noakes, M., \& Clifton, P. M. (2010). Renal Function Following Long-Term Weight Loss in Individuals with Abdominal Obesity on a Very-Low-Carbohydrate Diet vs High-Carbohydrate Diet. Journal of the American Dietetic Association, 110(4), 633-638.

Carneiro, G., Faria, A. N., Ribeiro Filho, F. F., Guimarães, A., Lerário, D., Ferreira, S. R. G., \& Zanella, M. T. (2003). Influência da distribuição da gordura corporal sobre a prevalência de hipertensão arterial e outros fatores de risco cardiovascular em indivíduos obesos. Revista da Associação Médica Brasileira, 49(3), 306-311.

D’Andrea Meira, I., Romão, T. T., Pires do Prado, H. J., Krüger, L. T., Pires, M. E. P., \& da Conceição, P. O. (2019). Ketogenic Diet and Epilepsy: What We Know So Far. Frontiers in Neuroscience, 13

Dehghan, M., Mente, A., Zhang, X., Swaminathan, S., Li, W., Mohan, V., \& Mohammadifard, N. (2017). Associations of fats and carbohydrate intake with cardiovascular disease and mortality in 18 countries from five continents (PURE): a prospective cohort study. The Lancet, 390(10107), $2050-2062$.

Enes, C. C. \& Slater, B. (2010). Obesidade na adolescência e seus principais fatores determinantes. Revista Brasileira de Epidemiologia, $13(1), 163-171$.

Faustino, B. E.; Braga de Azevedo; E., De Castro Silva, P. M., Sales, S. \& Barros, W. P. de S. (2011). Diabetes mellitus: busca ativa em portadores de obesidade. Cogitare Enfermagem, 16(1).

Gardner, C. D., Kiazand, A., Alhassan, S., Kim, S., Stafford, R. S., Balise, R. R., \& King, A. C. (2007). Comparison of the Atkins, Zone, Ornish, and LEARN Diets for Change in Weight and Related Risk Factors Among Overweight Premenopausal Women. JAMA, $297(9), 969$.

Gregory, R. M. (2016). A Low-Carbohydrate Ketogenic Diet Combined with 6-Weeks of Crossfit Training Improves Body Composition and Performance.

Hall, K. D., Guo, J., Courville, A. B., Boring, J., Brychta, R., Chen, K. Y., \& Walter, M. (2021). Effect of a plant-based, low-fat diet versus an animal-based, ketogenic diet on ad libitum energy intake. Nature Medicine, 27(2), 344-353.

Halyburton, A. K., Brinkworth, G. D., Wilson, C. J., Noakes, M., Buckley, J. D., Keogh, J. B., \& Clifton, P. M. (2007). Low- and high-carbohydrate weightloss diets have similar effects on mood but not cognitive performance. The American Journal of Clinical Nutrition, 86(3), 580-587.

Hashimoto, Y., Fukuda, T., Oyabu, C., Tanaka, M., Asano, M., Yamazaki., \& Fukui, M. (2016). Impact of low-carbohydrate diet on body composition: metaanalysis of randomized controlled studies. Obesity Reviews, 17(6), 499-509.

Hu, T., Yao, L., Reynolds, K., Niu, T., Li, S., Whelton, P. K., \& Bazzano, L. A. (2016). Adherence to low-carbohydrate and low-fat diets in relation to weight loss and cardiovascular risk factors. Obesity Science \& Practice, 2(1), 24-31.

Jabekk, P. T., Moe, I. A., Meen, H. D., Tomten, S. E., \& Høstmark, A. T. (2010). Resistance training in overweight women on a ketogenic diet conserved lean body mass while reducing body fat. Nutrition \& Metabolism, 7(1), 17.

Johnstone, A. M., Horgan, G. W., Murison, S. D., Bremner, D. M., \& Lobley, G. E. (2008). Effects of a high-protein ketogenic diet on hunger, appetite, and weight loss in obese men feeding ad libitum. The American Journal of Clinical Nutrition, 87(1), 44-55.

Johnstone, A. M., Lobley, G. E., Horgan, G. W., Bremner, D. M., Fyfe, C. L., Morrice, P. C., \& Duthie, G. G. (2011). Effects of a high-protein, low-carbohydrate v. high-protein, moderate-carbohydrate weight-loss diet on antioxidant status, endothelial markers and plasma indices of the cardiometabolic profile. British Journal of Nutrition, 106(2), 282-291. 
Kirk, S., Brehm, B., Saelens, B. E., Woo, J. G., Kissel, E., D’Alessio, D., \& Daniels, S. R. (2012). Role of Carbohydrate Modification in Weight Management among Obese Children: A Randomized Clinical Trial. The Journal of Pediatrics, 161(2), 320-327.e1.

Krebs, N. F., Gao, D., Gralla, J., Collins, J. S., \& Johnson, S. L. (2010). Efficacy and Safety of a High Protein, Low Carbohydrate Diet for Weight Loss in Severely Obese Adolescents. The Journal of Pediatrics, 157(2), 252-258.

Leith, W. (1961). Experiences with the Pennington diet in the management of obesity. Canadian Medical Association Journal, 84(25), 1411-1414.

Liu, X., Zhang, G., Ye, X., Li, H., Chen, X., Tang, L., \& Lin, X. (2013). Effects of a low-carbohydrate diet on weight loss and cardiometabolic profile in Chinese women: a randomised controlled feeding trial. British Journal of Nutrition, 110(8), 1444-1453.

Marchioni, D. M. L., Slater, B., \& Fisberg, R. M. (2004). Aplicação das Dietary Reference Intakes na avaliação da ingestão de nutrientes para indivíduos. Revista de Nutrição, 17(2), 207-216.

Mohorko, N., Černelič-Bizjak, M., Poklar-Vatovec, T., Grom, G., Kenig, S., Petelin, A., \& Jenko-Pražnikar, Z. (2019). Weight loss, improved physical performance, cognitive function, eating behavior, and metabolic profile in a 12-week ketogenic diet in obese adults. Nutrition Research, 62, 64-77.

National Academies of Sciences, Engineering, and Medicine (2019). Dietary Reference Intakes.

Noakes, T. D., \& Windt, J. (2017). Evidence that supports the prescription of low-carbohydrate high-fat diets: a narrative review. British Journal of Sports Medicine, 51(2), 133-139.

Paoli, A., Grimaldi, K., D’Agostino, D., Cenci, L., Moro, T., Bianco, A., \& Palma, A. (2012). International Society of Sports Nutrition, 9(1).

Paoli, A, Rubini, A., Volek, J. S., \& Grimaldi, K. A. (2013). Beyond weight loss: a review of the therapeutic uses of very-low-carbohydrate (ketogenic) diets. European Journal of Clinical Nutrition, 67(8), 789-796.

Paoli, A., Bianco, A., \& Grimaldi, K. A. (2015). The Ketogenic Diet and Sport. Exercise and Sport Sciences Reviews, 43(3), 153-162.

Partsalaki, , I., Karvela, A., \& Spiliotis, B. E. (2012). Metabolic impact of a ketogenic diet compared to a hypocaloric diet in obese children and adolescents. Journal of Pediatric Endocrinology and Metabolism, 25(7-8).

Penna, P. M.; Saron, M. L. G. \& Hermsdorff, H. H. M. (2020). O papel de adipocinas a relação entre obesidade e resistência à insulina: uma revisão integrativa. Cadernos UniFOA, 15(42).

Pereira, L. O, Francischi, R. P. de, \& Lancha Jr., A. H. (2003). Obesidade: hábitos nutricionais, sedentarismo e resistência à insulina. Arquivos Brasileiros de Endocrinologia \& Metabologia, 47(2), 111-127.

Philippi, S. T., (2015). Pirâmide dos alimentos: fundamentos básicos da nutrição. Editora Manole.

Pogozelski, W., Arpaia, N., \& Priore, S. (2005). The metabolic effects of low-carbohydrate diets and incorporation into a biochemistry course. Biochemistry and Molecular Biology Education, 33(2), 91-100.

Rhyu, H., \& Cho, S.-Y. (2014). The effect of weight loss by ketogenic diet on the body composition, performance-related physical fitness factors and cytokines of Taekwondo athletes. Journal of Exercise Rehabilitation, 10(5), 326-331.

Robergs, S. O. \& Roberts, R. A. (2002). Princípios fundamentais de fisiologia do exercício para aptidão, desempenho e saúde. Phorte.

Ruth, M. R., Port, A. M., Shah, M., Bourland, A. C., Istfan, N. W., Nelson, K. P., ... Apovian, C. M. (2013). Consuming a hypocaloric high fat low carbohydrate diet for 12 weeks lowers C-reactive protein, and raises serum adiponectin and high density lipoprotein-cholesterol in obese subjects. Metabolism, 62(12), 17791787.

Urbain, P., Strom, L., Morawski, L., Wehrle, A., Deibert, P., \& Bertz, H. (2017). Impact of a 6-week non-energy-restricted ketogenic diet on physical fitness, body composition and biochemical parameters in healthy adults. Nutrition \& Metabolism, 14(1).

Vargas, S., Romance, R., Petro, J. L., Bonilla, D. A., Galancho, I., Espinar, S., \& Benítez-Porres, J. (2018). Efficacy of ketogenic diet on body composition during resistance training in trained men: a randomized controlled trial. Journal of the International Society of Sports Nutrition, 15(1).

Yancy, W. S., Westman, E. C., McDuffie, J. R., Grambow, S. C., Jeffreys, A. S., Bolton, J., \& Oddone, E. Z. (2010). A Randomized Trial of a Low-Carbohydrate Diet vs Orlistat Plus a Low-Fat Diet for Weight Loss. Archives of Internal Medicine, 170(2), 136.

Weber, D. D., Aminzadeh-Gohari, S., Tulipan, J., Catalano, L., Feichtinger, R. G., \& Kofler, B. (2020). Ketogenic diet in the treatment of cancer- Where do we stand? Molecular Metabolism, 33, 102-121.

Wilson, J., \& Lowery, R., (2017). The Ketogenic Bible.

World Health Organization: WHO. (2020). Obesity. https://www.who.int/health-topics/obesity\#tab=tab_3

Zajac, A., Poprzecki, S., Maszczyk, A., Czuba, M., Michalczyk, M., \& Zydek, G. (2014). The Effects of a Ketogenic Diet on Exercise Metabolism and Physical Performance in Off-Road Cyclists. Nutrients, 6(7), 2493-2508.

Zinn, C., Wood, M., Williden, M., Chatterton, S., \& Maunder, E. (2017). Ketogenic diet benefits body composition and well-being but not performance in a pilot case study of New Zealand endurance athletes. Journal of the International Society of Sports Nutrition, 14(1). 